in flüssigem $\mathrm{NH}_{3}$. Es handelt sich also um einen neuen, bisher unbekannten Typ eines Carbonylhydrids, in dem der Wasserstoff keine Säurefunktion erfüllt, also nicht durch Metall ersetzt werden kann. Nach dem Abdunsten des $\mathrm{NH}_{3}$ bei $-33^{\circ}$ (Sdp. des $\mathrm{NH}_{3}$ ) erhält man das zinnoberrote Ammoniakat $\left[\mathrm{NiH}(\mathrm{CO})_{3}\right]_{2} \cdot 4 \mathrm{NH}_{3}$, das sich bei höheren Temperaturen unter stetiger $\mathrm{NH}_{3}$-Abgabe langsam zersetzt.

Das Nickel besitzt im $\left[\mathrm{NiH}(\mathrm{CO})_{3}\right]_{2}$ die Oxydationszahl - 1, was sich einwandfrei durch eine volumetrische Bestimmung des Wasserstoffs bei der Zersetzung mit Säure, die den Carbonylwasserstoff $\mathrm{zu} \mathrm{Ni}(\mathrm{CO})_{4}$ und $\mathrm{Ni}++$ oxydiert, beweisen läßt:

$$
\left.2 \underset{\mathrm{NiH}}{\mathrm{I}}(\mathrm{CO})_{3}\right]_{2}+2 \mathrm{H}^{+} \rightarrow 3 \stackrel{\circ}{\mathrm{o}} \mathrm{Ni}(\mathrm{CO})_{4}+\mathrm{Ni}^{++}+3 \mathrm{H}_{2} .
$$

Durch die analytische Bestimmung der bei der Säurezersetzung nach obiger Gleichung anfallenden Reaktionsprodukte wird die Zusammensetzung des Nickelcarbonylwasserstoffs zudem völlig sichergestellt.

Die dimere Struktur wurde durch Molekulargewichtsbestimmung nach der Methode der Dampfdruckerniedrigung in flüssigem Ammoniak ermittelt ${ }^{3}$. Es ergibt sich hiernach ein Molekulargewicht von 288 gemäß der Formel $\left[\mathrm{NiH}(\mathrm{CO})_{3}\right]_{2}$. Es sei an dieser Stelle nur darauf verwiesen, daß die Gruppen $\mathrm{Co}(\mathrm{CO})_{4}$ und $\mathrm{NiH}(\mathrm{CO})_{3}$ isoelektronisch sind, woraus sich der dimere Bau des Nickelcarbonylwasserstoffs konsequent erklären läßt.

Über weitere Eigenschaften und Reaktionen des Nickelcarbonylwasserstoffs sowie über dessen Strukturprobleme wird demnächst an anderer Stelle ausführlich berichtet werden.

3 A. Stock u. E. Pohland, Ber. dtsch. chem. Ges. 58, 657 [1925].

\section{Inden-Metallkomplexe des Kobalts}

Von E. O. Fischer, D. Seus und R. Jira

Anorganisch-chemisches Laboratorium der Techn. Hochschule München

(Z. Naturforschg. 8 b, 692-693 [1953]; eingeg. am 1. Okt. 1953)

Durch Umsetzung von Indenkalium mit $\mathrm{Co}\left(\mathrm{NH}_{3}\right)_{4}(\mathrm{SCN})_{2}$ in flüss. Ammoniak und Abbau des dabei ausfallenden $\left[\mathrm{Co}\left(\mathrm{NH}_{3}\right)_{6}\right]\left(\mathrm{C}_{9} \mathrm{H}_{7}\right)_{2}$ Ammoniak und Abbau des dabei ausfallenden $\left[\mathrm{Co}\left(\mathrm{NH}_{3}\right)_{6}\right]\left(\mathrm{C}_{9} \mathrm{H}_{7}\right)_{2}$
wurde schwarzes, paramagnetisches Di-indenyl-kobalt $\mathrm{Co}\left(\mathrm{C}_{9} \mathrm{H}_{7}\right)_{2}$ dargestellt. Bei der Oxydation desselben entsteht gelbes, diamagnetisches $\left[\mathrm{Co}\left(\mathrm{C}_{9} \mathrm{H}_{7}\right)_{2}\right]{ }^{+}-\mathrm{Kation}$, das sich außerdem auch durch oxydative Zersetzung einer Grignardlösung von $\mathrm{CoBr}_{2}$ und $\mathrm{C}_{9} \mathrm{H}_{7} \mathrm{MgBr}$ erhalten läßt. Beide Verbindungen stellen die ersten Glieder einer neuen, in Analogie zum Cyclopentadien zu erwartenden Komplexreihe des Indens mit Übergangsmetallen dar.

Beginnend mit der Entdeckung des Di-cyclopentadienyleisens $\mathrm{Fe}^{\mathrm{II}}\left(\mathrm{C}_{5} \mathrm{H}_{5}\right)_{2}{ }^{1}$, der Aufklärung ${ }^{2}$ und Bestätigung

1a T. J. Ke aly u. P. L. P a u s o n, Nature [London] 168, 1039 [1951].

1b S. A. Miller, J. A. Tebboth u. J. F. Tre$\mathrm{m}$ a in e, J. chem. Soc. [London] 632 [1952].

2a G. Wilkinson, M. Rosenblum, M. C. Whiting u. R. B. Woodward, J. Amer. chem. Soc. 74, 2125 [1952].

$2 b$ E. O. F is che r u. W. Pf a b, Z. Naturforschg. 7 b, 377 [1952]. seiner ungewöhnlichen Molekülstruktur durch Röntgenanalyse 2 b, 3 steht seither das System dieser neuartigen Organo-Metallkomplexe rasch ím weiteren Ausbau. Entsprechende flüchtige Verbindungen wie $\mathrm{RuII}\left(\mathrm{C}_{5} \mathrm{H}_{5}\right)_{2}{ }^{4}$, $\left.\mathrm{NiII}\left(\mathrm{C}_{5} \mathrm{H}_{5}\right)_{2}{ }^{5}, \mathrm{CoII}_{(} \mathrm{C}_{5} \mathrm{H}_{5}\right)_{2}{ }^{6}, \mathrm{Cr}^{\mathrm{II}}\left(\mathrm{C}_{5} \mathrm{H}_{5}\right)_{2}{ }^{7}, \mathrm{MnII}\left(\mathrm{C}_{5} \mathrm{H}_{5}\right)_{2}{ }^{7}$ sowie zahlreiche weitere Komplexe desselben Bautyps mit FeIII 2, CoIII 2b, 8, NiIII 5, TiIII, IV 5a, VIV 5a, ZrIV 5a, RhIII 9, IrIII 9 konnten dargestellt werden. Auf Grund röntgenographischer Untersuchungen an $\mathrm{Fe}\left(\mathrm{C}_{5} \mathrm{H}_{5}\right)_{2}{ }^{2 b}, 3$, $\mathrm{Ni}\left(\mathrm{C}_{5} \mathrm{H}_{5}\right)_{2}{ }^{5 \mathrm{~b}}, \mathrm{Co}\left(\mathrm{C}_{5} \mathrm{H}_{5}\right)_{2}{ }^{6}, \mathrm{Cr}\left(\mathrm{C}_{5} \mathrm{H}_{5}\right)_{2}{ }^{7}$ ist übereinstimmend bei all diesen Verbindungen das jeweilige Übergangsmetall zentral zwischen den beiden antiprismatisch parallel einander gegenüberstehenden Cyclopentadienylanionen eingelagert anzunehmen. Magnetische Untersuchungen zeigen, daß die Bindung des Zentralatoms über die $\pi$-Elektronen der aromatisierten Ringe erfolgt.

Angesichts der außergewöhnlichen Bindungsbetätigung des Cyclopentadiens gegenüber Übergangselementen war bereits vor einiger Zeit die Frage aufgegriffen worden, inwieweit vornehmlich auch das homologe Inden einer ähnlichen Komplexbildung zugänglich ist. Versuche in dieser Richtung, die inzwischen bei Kobalt mit der im folgenden kurz beschriebenen Darstellung eines ungeladenen Di-indenyl-kobalt(II) $\mathrm{Co}\left(\mathrm{C}_{9} \mathrm{H}_{7}\right)_{2}$ wie eines geladenen Di-indenyl-kobalt(III)-Kations $\left[\mathrm{Co}\left(\mathrm{C}_{9} \mathrm{H}_{7}\right)_{2}\right]+$ zu einem gewissen Abschluß gelangt sind, lassen bereits jetzt den Schluß zu, daß eine dem System der CyclopentadienKomplexe homologe Reihe der Inden-Komplexe zugänglich sein wird.

Zur Darstellung von $\mathrm{Co}\left(\mathrm{C}_{9} \mathrm{H}_{7}\right)_{2}$ hat sich wiederum das von uns an den Cyclopentadien-Verbindungen entwickelte Verfahren in flüssigem Ammoniak bewährt. Durch Umsetzung von metallischem Kalium mit Inden wurde zunächst das im Ammonosystem lösliche Kaliumsalz des Indenyl-anions erhalten. Letzteres läßt sich alsdann gemäß:

$\mathrm{Co}\left(\mathrm{NH}_{3}\right)_{4}(\mathrm{SCN})_{2}+2 \mathrm{KC}_{9} \mathrm{H}_{\bar{\tau}} \stackrel{\mathrm{NH}_{3} \mathrm{fl} .}{\longrightarrow}\left[\mathrm{Co}\left(\mathrm{NH}_{3}\right)_{6}\right]\left(\mathrm{C}_{9} \mathrm{H}_{\overline{7}}\right)_{2}+2 \mathrm{KSCN}$,

als schwerlösliches, gelbbraunes $\left[\mathrm{Co}\left(\mathrm{NH}_{3}\right)_{6}\right]\left(\mathrm{C}_{9} \mathrm{H}_{7}\right)_{2}$ ausfällen. Im Vakuum spaltet diese Hexammin-Verbindung unter rascher Farbvertiefung nach Schwarzbraun $\mathrm{NH}_{3}$ ab. Extrahiert man den Rückstand unter Luftabschluß mit Petroläther und engt die filtrierte, tief braunrote Lö-

3a P. F. Eil and u. R. P e pins ky, J. Amer. chem. Soc. 74, 4971 [1952].

3b J. D. Dunitz u. L. E. Orgel, Nature [London] 171, 121 [1953].

${ }^{4}$ G. Wilkins o n, J. Amer. chem. Soc. 74, 6146 [1952].

5a G. Wilkinson, P. L. Paus on, J. M. B ir mingh am, F. A. Cotton, ibid. 75, 1011 [1953].

5 b E. O. Fi s che r u. R. Jira, Z. Naturforschg. 8b. 217 [1953].

6 E. O. F is che r u. R. J i r a, ibid. 8 b, 327 [1953].

7 E. O. Fischer u. W. Hafner, ibid. 8 b, 444 [1953].

8 a G. Wilk ins o n, J. Amer. chem. Soc. 74, 6148 [1952].

$8 \mathrm{~b}$ E. O. F is cher u. R. Jir a, Z. Naturforschg. 8 b, 1 [1953].

9 F. A. Cotton, R. O. Whipple u. G. Wilk in s o n, J. Amer. chem. Soc. 75, 3586 [1953]. 
sung ein, so kristallisieren glitzernde, schwarze Kristalle aus. Ihre Analyse zeigt, daß Di-indenyl-kobalt $\mathrm{Co}\left(\mathrm{C}_{9} \mathrm{H}_{7}\right)_{2}$ vorliegt.

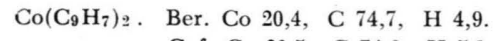

Di-indenyl-kobalt ist mit braunroter Farbe in organischen Lösungsmitteln wie Benzol, Äther oder Alkohol sehr gut, in Petroläther etwas weniger löslich und bei absolutem Sauerstoffausschluß darin völlig beständig. In $\mathrm{N}_{2}$-gesättigtem $\mathrm{H}_{2} \mathrm{O}$ bleibt es ungelöst und unangegriffen. Es zeigt unter Stickstoff bei etwa $160^{\circ}$ langsames Sintern, bei $178^{\circ}$ bis $181^{\circ}$ einen wenig scharfen Schmelzpunkt. Im Hochvakuum erfolgt Sublimation ab $100^{\circ}$ bis $120^{\circ}$. Die Oxydationsempfindlichkeit ist gegenüber $\mathrm{Co}\left(\mathrm{C}_{5} \mathrm{H}_{5}\right)_{2}$ merklich geringer. Mittels der kryoskopischen Methode wurde in Benzol ein M.G. von 304 (ber. 289,0) gefunden. Eine Untersuchung der Lichtabsorption in Petroläther ergab Maxima bei $410 \mathrm{~m} \mu, 322 \mathrm{~m} \mu, 274 \mathrm{~m} \mu$ sowie Minima bei $365 \mathrm{~m} \mu$ und $294 \mathrm{~m} \mu$.

Bei Behandlung mit Oxydationsmitteln wie $\mathrm{H}_{2} \mathrm{O}_{2}$ oder $\mathrm{K}_{2} \mathrm{~S}_{2} \mathrm{O}_{8}$ in wässeriger Lösung geht $\mathrm{Co}\left(\mathrm{C}_{9} \mathrm{H}_{7}\right)_{2}$ in ein tiefgelbes, sehr beständiges $\left[\mathrm{Co}\left(\mathrm{C}_{9} \mathrm{H}_{7}\right)_{2}\right]+$-Kation über. Unmittelbar läßt sich dasselbe in einfacher Weise auch durch Grignardierung von $\mathrm{CoBr}_{2}$ mit $\mathrm{C}_{9} \mathrm{H}_{7} \mathrm{MgBr}$ in Äther und anschließende oxydative Zersetzung mit Eis und $\mathrm{H}_{2} \mathrm{O}_{2}$ erhalten. Es gleicht in seiner Stabilität wie seinen Fällungsreaktionen weitgehend dem entspr. $\left[\mathrm{Co}\left(\mathrm{C}_{5} \mathrm{H}_{5}\right)_{2}\right]+$ Kation. So fällen Reineckeat und Phenyloborat gleichfalls schwerlösliches gelbes $\left[\mathrm{Co}\left(\mathrm{C}_{9} \mathrm{H}_{7}\right)_{2}\right]\left[\mathrm{Cr}(\mathrm{SCN})_{4}\left(\mathrm{NH}_{3}\right)_{2}\right]$ und gelbbraunes $\left[\mathrm{Co}\left(\mathrm{C}_{9} \mathrm{H}_{7}\right)_{2}\right]\left[\mathrm{B}\left(\mathrm{C}_{6} \mathrm{H}_{5}\right)_{4}\right]$. Perchlorat ergibt ein in feinen, orangebraunen Nadeln kristallisierendes, mäßig lösliches $\left[\mathrm{Co}\left(\mathrm{C}_{9} \mathrm{H}_{7}\right)_{2}\right] \mathrm{ClO}_{4}$. Auch mit $\mathrm{PF}_{6}^{-}$, $\mathrm{J}_{3}^{-}, \mathrm{Br}_{3}{ }^{-}, \mathrm{BiJ}_{4}^{-},\left[\mathrm{Co}(\mathrm{CO})_{4}\right]^{-}$erhält man wiederum schwerlösliche Niederschläge, während die Salze mit den üblichen Anionen wie $\mathrm{SO}_{4}^{-2}, \mathrm{Cl}^{-}, \mathrm{Br}^{-}, \mathrm{J}^{-}, \mathrm{NO}_{3}^{-}, \mathrm{CN}^{-}, \mathrm{CO}_{3}^{-2}, \mathrm{PO}_{4}^{-3}$ leichtlöslich sind. Die freie Base ist in wässeriger Lösung beständig. Eine Messung der Lichtabsorption von $\left[\mathrm{Co}\left(\mathrm{C}_{9} \mathrm{H}_{7}\right)_{2}\right] \mathrm{ClO}_{4}$ in $\mathrm{H}_{2} \mathrm{O}$ ergab ein charakteristisches Maximum bei $322 \mathrm{~m} \mu$ sowie ein Minimum bei $268 \mathrm{~m} \mu$.

Entscheidende Bedeutung kommt dem Ergebnis der magnetischen Messungen zu. $\mathrm{Co}\left(\mathrm{C}_{9} \mathrm{H}_{7}\right)_{2}$ zeigt Paramagnetismus. Es wurde hierfür eine molare Suszeptibilität von $\chi_{\mathrm{Mol}}^{29{ }^{\circ} \mathrm{K}}=+1180 \cdot 10^{-6} \mathrm{~cm}^{3} / \mathrm{Mol}$ entspr. einem effektiven magnetischen Moment von 1,76 Bohrschen Magnetonen gefunden. Dies entspricht dem Vorliegen eines ungepaarten Elektrons. Eine Messung des Kations $\left[\mathrm{Co}\left(\mathrm{C}_{9} \mathrm{H}_{7}\right)_{2}\right]+$ als Perchlorat ergab demgegenüber Diamagnetismus. Es

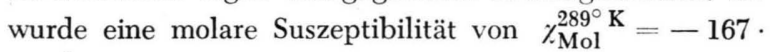
$10^{-6} \mathrm{~cm}^{3} / \mathrm{Mol}$ ermittelt.

Angesichts dieser mit den magnetischen Befunden der entsprechenden Cyclopentadien-Komplexe $\mathrm{Co}\left(\mathrm{C}_{5} \mathrm{H}_{5}\right)_{2}$ und $\left[\mathrm{Co}\left(\mathrm{C}_{5} \mathrm{H}_{5}\right)_{2}\right]+$ völlig übereinstimmenden Verhältnisse ist unseres Erachtens vorläufig der Schluß berechtigt, daß in ähnlicher Weise wie bei jenen die $\pi$-Elektronen des aromatisierten Fünfrings die Bindung mit dem zentralen Metall bewirken. Für die Gestalt des Moleküls bzw. Ions folgt alsdann, daß bei $\mathrm{Co}\left(\mathrm{C}_{9} \mathrm{H}_{7}\right)_{2}$ zentrales Kobalt(II), beim Kation hingegen zentrales Kobalt(III) zwischen den antiprismatisch parallel einander gegenüberstehenden
Fünfringen der beiden Indenyl-anionen eingelagert sein sollte, während deren anellierte Benzolringe in transStellung stehen dürften. Abb. 1 zeigt diesen Strukturvorschlag.

Eine sichere Aussage über die Elektronen-Konfiguration des $\mathrm{Co}(\mathrm{II})$ und $\mathrm{Co}(\mathrm{III})$ und die Bindungsverhältnisse erscheint als verfrüht. Der Gedanke einer Auffüllung des Kobalts zur Krypton-Konfiguration, z. B. durch BindungsBetätigung der je drei $\pi$-Elektronenpaare beider Fünfringe, liegt beim Kation jedoch sehr nahe. Im Falle der leicht oxydierbaren ungeladenen Verbindung wäre alsdann zusätzlich ein 4d- bzw. 5s-Elektron anzunehmen.

In charakteristischer Weise unterscheiden sich von den hier beschriebenen Komplexen des Kobalts im übrigen die

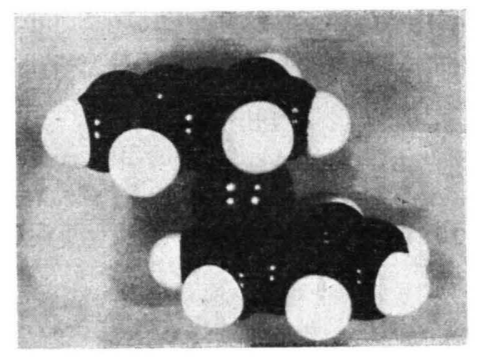

Abb. 1. Strukturmodell von $\mathrm{Co}\left(\mathrm{C}_{9} \mathrm{H}_{7}\right)_{2}$.

kürzlich erstmals dargestellten metallorganischen IndenVerbindungen des Zinns ${ }^{10}$. Bereits die Existenz eines Tetra-indenyl-zinns $\mathrm{Sn}\left(\mathrm{C}_{9} \mathrm{H}_{7}\right)_{4}$ zeigt, daß hier entsprechend den Eigenschaften des Metalls als Hauptgruppen-Element keine Bindung über $\pi$-Elektronen und keine „Doppelkegel-Struktur“ 11 mehr vorliegen dürfte. Metallorganische Durchdringungs-Komplexe vom Typ der obigen KobaltVerbindungen, deren allgemeine Formel $\left[\mathrm{Mex}\left(\mathrm{C}_{9} \mathrm{H}_{7}\right)_{2}\right]^{-2}$ ( $\mathrm{x}=$ Wertigkeit) den entsprechenden CyclopentadienVerbindungen der Zusammensetzung $\left[\mathrm{Mex}\left(\mathrm{C}_{5} \mathrm{H}_{5}\right)_{2}\right]^{-2}$ entspricht, werden wiederum nur bei Übergangs-Metallen zu erwarten sein. Ein weiterer Hinweis hierfür ergab sich inzwischen auch durch die Darstellung eines tiefrotbraunen Di-indenyl-nickels.

$\mathrm{Ni}\left(\mathrm{C}_{9} \mathrm{H}_{7}\right)_{2}$. Ber. Ni 20,3, C 74,7, H 4,9. Gef. Ni 20,4 , C 74,4 , H 5,1 .

Die Untersuchüng der mit kirschroter Farbe in organischen Mitteln löslichen Verbindung wie überhaupt die naheliegende Erweiterung auf andere Übergangs-Metalle ist im Gange.

Wir danken an dieser Stelle Herrn Prof. Dr. W. H i eber für die großzüsige Unterstützung mit Institutsmitteln sowie den Herren Dipl.-Phys. F. En gelmann und cand. phys. W. Le chner, Physikalisches Institut der T.H. München, für die Durchführung der magnetischen Messungen.

10 H. Zimmer u. H. W. Sparmann, Naturwiss. 40, 220 [1953].

11 Von G. Wilkins on u. Mitarb. als „sandwichstructure" bezeichnet. 